\title{
Therapeutic options in pediatric non alcoholic fatty liver disease: current status and future directions
}

\author{
Pietro Vajro ${ }^{1 *}$, Selvaggia Lenta ${ }^{2}$, Claudio Pignata ${ }^{2}$, Mariacarolina Salerno $^{2}$, Roberta D'Aniello ${ }^{1}$, Ida De Micco ${ }^{2}$, \\ Giulia Paolella ${ }^{1}$ and Giancarlo Parenti ${ }^{2}$
}

\begin{abstract}
The epidemics of overweight and obesity has resulted in a significant increase of non alcoholic fatty liver disease (NAFLD), a potentially progressive condition. Currently, obesity related hepatopathy represents therefore the main cause of pediatric chronic liver disease. The first choice treatment at all ages is weight loss and/or lifestyle changes, however compliance is very poor and a pharmacological approach has become necessary. In the present article we present a systematic literature review focusing on established pediatric NALFD drugs (ursodeoxycholic acid, insulin sensitizers, and antioxidants) and on innovative therapeutic options as well.

Regarding the former ones, a pediatric pilot study highlighted that ursodeoxycholic acid is not efficient on transaminases levels and bright liver. Similarly, a recent large scale, multicenter randomized clinical trial (TONIC study) showed that also insulin sensitizers and antioxidant vitamin E have scarce effects on serum transaminase levels. Among a large series of novel therapeutic approaches acting on recently proposed different pathomechanisms, probiotics seem hitherto the most interesting and reasonable option for their safety and tolerability. Toll-like receptors modifiers, Pentoxifylline, and Farnesoid $X$ receptors agonists have been still poorly investigated, and will need further studies before becoming possible promising innovative therapeutic strategies.
\end{abstract}

Keywords: Non alcoholic fatty liver disease, Children, Therapy

\section{Introduction}

Non alcoholic fatty liver disease is nowadays the most common cause of chronic liver disease also in pediatric age, as a result of the increasing prevalence of childhood obesity. It represents a spectrum of liver diseases ranging from simple steatosis to steatohepatitis, with, in some cases, possible fibro/cirrhotic progression, thus increasing liver-related morbidity and mortality [1]. The mainstay of NAFLD therapy is represented by lifestyle interventions on obesogenic environment and sedentary life, which aim to improve obesity, obesity-related hepatic changes, and quality of life as well [2]. Unfortunately this target is difficult to be achieved, and results are unsatisfactory. In fact, only a little proportion of individuals is really able to steadily lose weight and to practice

\footnotetext{
*Correspondence: pvajro@unisa.it

'Chair of Pediatrics, Medical School of the University of Salerno, Salerno, Italy, and ELFID, Naples, Italy

Full list of author information is available at the end of the article
}

physical exercises [1]. No agreement exists on NAFLD management of obese children who result not-compliant to prescriptions. Since several established pathogenetic mechanisms (in particular insulin resistance, oxidative stress, and apoptosis) seem to be involved in NAFLD, a number of therapeutic agents targeting these mechanisms (Figure 1) have been tested not only in animal models but also in human adults and in children. Results however are still puzzling and/or unsatisfactory [1,3].

The aim of this article is to review current NAFLD treatments and possible novel options in the pediatric age group, by providing recent evidences from literature.

\section{Materials and methods}

Search strategy and inclusion criteria

Through the MEDLINE database we searched for articles on NAFLD treatment in pediatric age appearing from $1^{\text {st }}$ May 2009 (date of publication of the meta-analysis of Socha et al. [3]) to $1^{\text {st }}$ August 2012. No language 


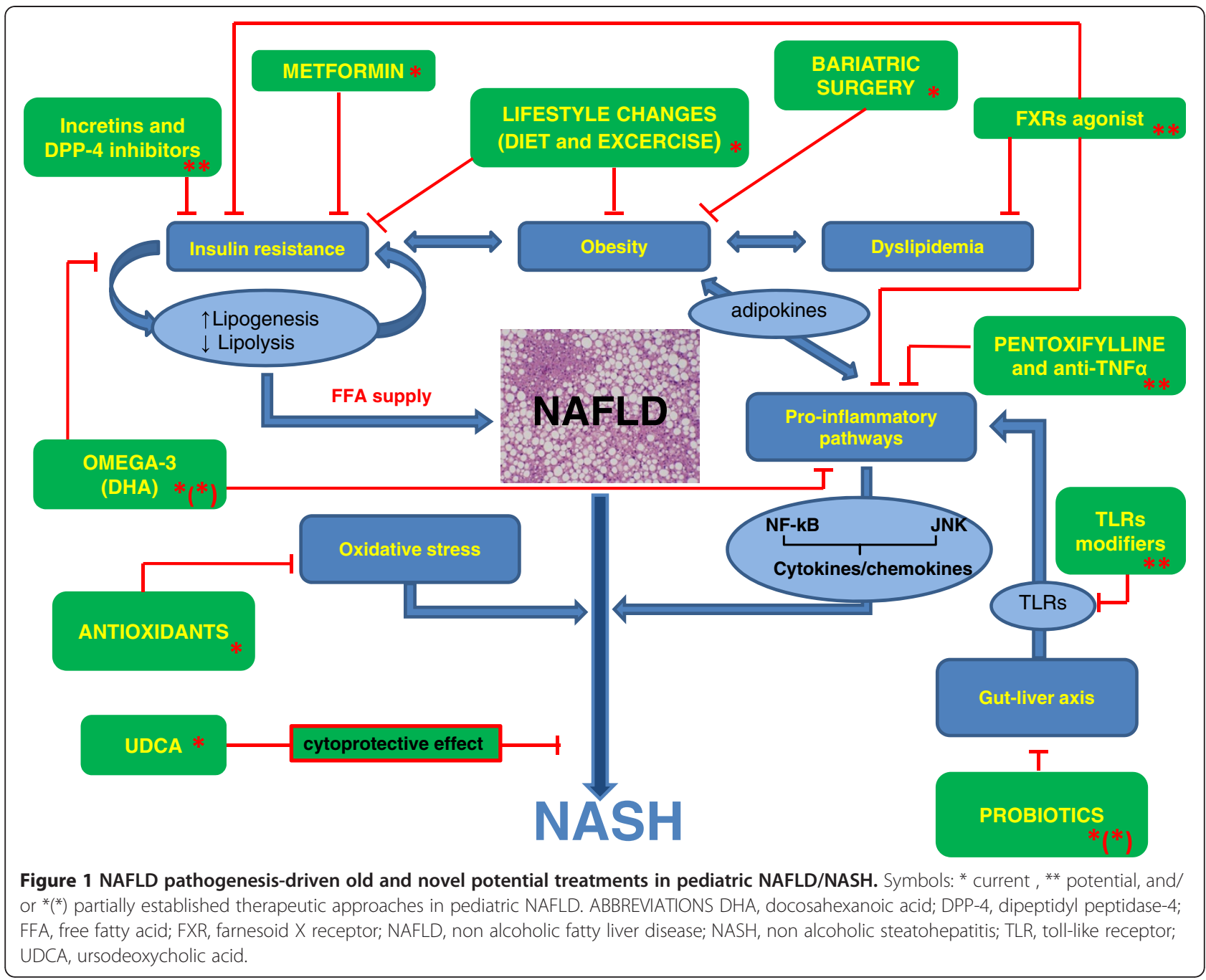

restrictions were imposed. Eligible studies and research articles on NAFLD therapy in pediatric age were reviewed. When paucity/absence of pediatric data were evident we retrieved also articles on NAFLD therapy in adults. The electronic literature search was performed using the following keywords which had been suggested by previous manual search: NAFLD, NASH, fatty liver, treatment and/or therapy, lifestyle interventions, weight loss, physical activity, antioxidant and/or vitamin E, metformin and/or insulin sesitizers, ursodeoxycholic acid, probiotics and prebiotics, omega-3-fatty acid and/ or docosahexaenoic acid, bariatric surgery, toll like receptors modifiers, pentoxifylline, farnesoid X receptor agonist, incretin mimetics, dipeptidyl peptidase-4 inhibitors, angiotensin-converting enzyme (ACE) inhibitors, angiotensin-receptor blockers (ARBs), children, adults, animal model. Title and abstract of each retrieved reference was screened by one author. Full papers were screened independently by two researchers. Discrepancies between reviewers were resolved by consensus. A narrative review of novel and old therapeutic strategies was conducted.

\section{Data extraction}

Data were collected by using a data extraction form. Studies were collected for old/present and novel/ potential pharmacological strategies both in children and adults and/or in animal models.

\section{Results}

\section{Lifestyle interventions}

\section{Weight loss}

Weight reduction in NAFLD patients reduces the delivery of free fatty acids (FFA) to the liver, improving extrahepatic insulin sensitivity by means of a better peripheral glucose utilization. Furthermore it promotes a reduction of reactive oxygen substances (ROS) and adipose tissue inflammation [4].

Recent studies confirmed an amelioration in transaminases levels and in several metabolic parameters (lipids, 
fasting glucose and insulin sensitivity indices) also in pediatric age [5-8].

A sucrose-rich diet (e.g. soft-drinks) increases the hepatic synthesis of triglycerides: rats and humans that are fed either sucrose or fructose enriched diets develop fatty and fibrotic liver. Fructose reduction may decrease insulin resistance (IR) and lipogenesis, and also hepatic pro-inflammatory/fibrogenetic effects [9].

Other helpful proposed dietetic measures include the reduction of dietary intake of satured/trans fat, increased intake of fibers [10] and a higher intake of polyunsaturated fat (omega-3) [11].

Weight loss (target= approximately $5-10 \%$ of basal weight) should be achieved gradually, since extreme slimming diets may lead to the onset of severe metabolic disorders and promote liver histological damage. Dietary interventions are still not based on precise evidence-based guidelines: in childhood however diets must be balanced to allow a healthy and harmonic growth [12]. The ESPGHAN Committee on Nutrition [13] suggests that energy intake should be individually determined, and slowly rather than rapidly absorbed carbohydrates should be preferred. A clinical approach in older children should consist in a weight loss of about $500 \mathrm{~g} /$ week. In younger children one might suggest even to simply not gain further weight in order to improve the weight-height ratio, and also to improve waist circumference (i.e. the most sensitive marker of visceral obesity and its related liver involvement) $[7,14]$.

\section{Physical activity}

Physical activity should be integrated in NAFLD therapy because of its beneficial effect independent of weight loss, by enhancing insulin sensitivity and glucose homeostasis [15]. Exercise has a beneficial effect also on FFA metabolism, by enhancing whole-body lipid oxidation [16] and decreasing hepatic triglyceride accumulation [17].

In obese adolescents a three-month resistance exercise program, which involved all major muscle groups $2 \times 1$ $\mathrm{h} /$ week, resulted in significant strength and lean body mass gain. Although hepatic fat content remained fairly stable, hepatic insulin sensitivity increased and glucose production rate decreased, without weight loss [18]. Despite aerobic exercise seems to have more extensive effects, a longer duration and/or a more intensive resistance exercise program may be required for reduction of hepatic fat content. For those who have physical limitations and/or low motivation that prevent them from performance of aerobic activity, resistance exercise can serve as an alternative option.

Lifestyle changes should include all family members to increase compliance, along with a multidisciplinary approach, including childhood dedicated dietician and psychologist. Pediatricians and pediatric gastroenterohepatologists should have a leading role in the case management of pediatric NAFLD, working with other professional specialties involved in physical and intellectual growth [9].

Since treatment options are nowadays still limited, obesity prevention should still be the optimal strategy in the management of NAFLD. In view of the poor adherence to diets and exercise, novel strategies have also been envisaged, including "e-health platforms". These are aimed at improving treatment compliance and promoting the mechanisms of patients self-control, to obtain weight loss maintenance, and to prevent relapse by establishing healthy lifestyle habits [19].

\section{Drug treatments for NAFLD in pediatric age}

When diet and/or exercise are not obtainable, a pharmacological approach acting upon one or more specific targets involved in NAFLD aetiopathogenesis should be used (Figure 1). Insulin sensitizers, antioxidants and cytoprotective agents are the drugs which have hitherto been more extensively used.

\section{Insulin sensitizers}

Hepatic insulin resistance has been highlighted in most of the children/adolescents with NAFLD [20]. Even if the molecular mechanism is poorly understood, insulin resistance has a key role in NAFLD by promoting the storage of FFA. For this reason it might be considered an advantageous therapeutic target [1]. The only insulinsensitizer which has been well evaluated in pediatric NAFLD is metformin. A pilot study in 10 non diabetic children with NASH showed improvement of fatty liver at Magnetic Resonance Spectroscopy, and reduction of ALT level [21]. Subsequent studies however yielded divergent results [22]. The most recent TONIC study [a large, multicenter RCT evaluating the effect of a 2 year therapy with Vitamin E (400 IU twice daily) or insulin sensitizer metformin (500 mg twice daily) or placebo in 173 children with biopsy-confirmed NAFLD] substantiated its scarce effectiveness in lowering serum ALT, with only marginal effects on hepatic histology [23].

\section{Antioxidants}

Oxidative stress is considered another major contributor to the progression from simple steatosis to NASH by protecting susceptible components of biological membranes from lipid peroxidation. Alpha-tocopherol (vitamin E) is the more extensively studied antioxidant in pediatric NAFLD. In an open label pediatric trial Lavine et colleagues found that Vitamin E (400-1.200 IU/day) may induce a decrease of serum transaminases levels, which was not related to a reduction in bright liver at ultrasonography and BMI values [24]. Other studies comparing the effect of Vitamin E [25,26] \pm ascorbic acid [27] vs. weight loss alone, however showed that antioxidant treatment 
was not more efficient than exclusive lifestyle changes. In the double blind RCT study TONIC [23], Vitamin E treatment did not attain a significant and sustained decrease in ALT levels compared to placebo. However it was better than placebo in inducing resolution of an histologically borderline or defined NASH, and in improving hepatocellular ballooning and NAFLD activity histological score. Cysteamine bitartrate is a recently described potent antioxidant which has been hitherto used only in a small pediatric NAFLD pilot study with promising effects on reduction of transaminase levels [28]. It is currently under evaluation in a multicenter placebo-controlled clinical trial (Cysteamine Bitartrate Delayed-Release for the Treatment of NAFLD in Children) [29].

Other newer antioxidants have shown to represent potential therapeutic tools but have been studied only in adult NAFLD [1].

\section{Hepatoprotective agents}

The hydrophilic bile acid Ursodeoxycholic acid (UDCA) might antagonize the progression of NAFLD/NASH, through protection of hepatocytes from bile saltsmediated mitochondrial injury, antiapoptotic signaling pathway, and immunomodulatory function [30]. However, conventional doses of UDCA used in a pilot pediatric RCT were not effective on ALT levels and ultrasonographic liver abnormalities [31]. These data have been confirmed also in several adults studies [32]. Presently it is under verification if (potentially risky [33]) higher doses of UDCA may be necessary [1].

\section{Innovative drugs}

Summing up, ordinary NAFLD therapeutic approaches reviewed above appear most often unsatisfactory or inadequate both in adults and in children. Table 1 summarizes a series of newer treatment targets which have been partially explored in preliminary animal and/or patients studies and may therefore deserve attention for more extensive upcoming investigation in adult and pediatric NAFLD as well.

\section{Probiotics and prebiotics}

A growing body of evidence [37] shows that the gut microbiota controls obesity and visceral fat storage. Specific variations in gut microbiota in early life may

Table 1 Still not conventional NAFLD treatment targets

\begin{tabular}{ll}
\hline Therapeutic target & Pharmacolological treatment \\
\hline Gut-liver axis & Probiotics [34] \\
Dyslipidemia/Insulin & Omega-3 DHA (docosahexaenoic acid) \\
Resistance & {$[12]$} \\
TNF-a pathway & Pentoxifylline and anti-TNF-a [35] \\
FXRs pathway & Agonist of the farnesoid X receptor \\
& (FXR) [36] \\
\hline
\end{tabular}

determine a major risk factor of obesity and its complications later in life [38]. Small intestinal bacterial overgrowth (SIBO) (a frequent condition in obese individuals, mainly prompted by slowing of the oro-coecal transit time) may promote NAFLD progression to nonalcoholic steatohepatitis by enhancing intestinal permeability and by favoring absorption of endotoxins with pro-inflammatory and pro-fibrogenetic effects on the liver [39].

Probiotics are live microorganisms which when consumed in adequate amounts, confer an healthy benefit to the host [40]. Gut microbiota manipulation with probiotics in rodents with fatty liver reduces intestinal inflammation and improves the epithelial barrier function $[41,42]$. Therefore, probiotics could represent a new effective treatment also in NAFLD human patients. Loguercio and colleagues have shown that probiotics may reduce NAFLD liver injury and may improve liver function tests [43]. The Cochrane review [44] and a subsequent pediatric meta-analysis [3] however have highlighted that probiotics treatment in patients with NAFLD and non alcoholic steatohepatitis could not be recommended because of the lack of randomized clinical trials. More recently a double-blind RCT showed that the treatment with 500 million of Lactobacillus bulgaricus and Streptococcus thermophiles/day in adults with biopsyproven NAFLD causes a reduction of liver transaminases level [45]. In children, another double-blind RCT study also showed that obese patients with NAFLD (mean age 10.7 years), treated with Lactobacillus GG (12 billion CFU/day for 8 weeks), irrespective of changes in BMI $\mathrm{z}$ score and visceral fat, reached a significant decrease (up to normalization in $80 \%$ of cases) in alanine aminotransferase and in antipeptidoglycan-polysaccharide antibodies (a SIBO marker). TNF- $\alpha$ serum levels, and bright liver parameters remained quite stable [34].

Prebiotics are non digestible food ingredients that beneficially affect the host by selectively stimulating growth and/or modifying metabolic activity of selected intestinal bacteria [40]. By reducing the risk of obesity and altering the composition of gut microbiota, prebiotics may represent therefore another potential therapeutic approach. Studies in obese rats have shown that prebiotic fibers improve or normalize gut microbiota dysbiosis by increasing Firmicutes and decreasing Bacteroidetes phylae [46]. Gut microbiota modulation is correlated with an improvement of glucose, energy intake, insulin, satiety hormones, hepatic cholesterol, and triglyceride accumulation [45].

\section{Omega-3 long chain polyunsaturated fatty acids}

Polyunsaturated fatty acids (PUFAs) class is present in several natural foods and includes "essential fatty acids" like $\omega-3$ and $\omega-6$ acids that the body needs but cannot 
produce. The balance between dietary $\omega-3$ and $\omega-6$ strongly affects several functions of PUFAs. Recent pharmacological studies in NAFLD animal models and in adult humans focusing on the effect of oral treatment with $\omega-3$ fatty acids, demonstrate that they have both anti-inflammatory and insulin sensitizing properties, suggesting a potential role in treatment of NAFLD [47]. In NAFLD children $\omega 3$-docosahexaenoic acid treatment for 6 months improved ultrasonographic fatty liver and insulin sensitivity [12]. Because this treatment is well tolerated in pediatric population, DHA deserve further studies in the management of children with NAFLD.

\section{A surgical approach: bariatric surgery}

Bariatric surgery may be proposed for a durable weight loss in individuals with severe obesity. A study in young adults (median age $=18.6$ years) showed that bariatric surgery, by causing significant weight loss, leads to an improvement in clinical and hepatic histological parameters [48]. The guidelines for younger ages however are not yet fully standardized, and studies on safety and long term efficacy are therefore needed in developing and transition age [49].

\section{Other potential novel candidate therapeutic targets}

A series of other interesting approaches, hitherto explored only in NAFLD animal models or in few pilot studies in adults will possibly become in future the object of study in pediatric population, as well (Table 1).

\section{TNF-a pathway antagonists}

TNF- $\alpha$ and other adipocytokines produced by adipose tissue are involved in NAFLD progression. Pentoxifylline, a phosphodiesterase inhibitor, exerts immunomodulatory functions by antagonizing the TNF- $\alpha$ pathway. In adults with $\mathrm{NASH}$, pentoxifylline treatment showed good tolerability and could decrease serum ALT levels and improve histological features, as well [35].

\section{Farnesoid $\mathrm{X}$ receptor (FXR) agonist}

The nuclear bile acid receptor FXR, strongly expressed in bowel and liver, is probably involved in NAFLD pathogenesis, by mediating control of lipids and glucose homeostasis, and controlling bacterial flora growth. Altogether, these effects may induce reduction of hepatic inflammation and fibrogenesis, through different mechanisms. Therefore, recently developed FXR agonists have a potential role in the pharmacological therapy of NAFLD/NASH [36].

\section{Toll-like receptor (TLR) modifiers}

TLRs are receptors sensing microbial components of gut microbiota. A number of recent evidences suggests the role of SIBO and increased intestinal permeability in
NAFLD, by exposing -via portal vein- the liver to an high load of intestinal noxae including lipopolysaccharide and other pathogen-associated molecular patterns [50]. Furthermore, TLRs stimulation causes downstream activation of the inflammatory response. Pro-inflammatory patterns result in production of cytokines and chemokines implicated in progression from simple steatosis to steatohepatitis and fibro-cirrhosis; so therapeutic manipulation of innate immune system through TLRs modifiers, formerly evaluated for autoimmune diseases [51], might be a new potential therapeutic target for pediatric NAFLD, but further studies are necessary.

\section{Incretin mimetics and DPP-4 (dipeptidyl peptidase-4) inhibitors}

Glucagon-like peptid-1 (GLP-1) is an incretin secreted in response to food intake, allotted to multiple functions, including, the stimulation of glucose-dependent insulin secretion and inhibition of glucagon release. The enzyme DPP-4 rapidly degrades circulating GLP-1 (half-life: 1-2 minutes). Recent animal model and NAFLD adults studies showed an effective role of GLP-1 receptor agonists resistent to DPP-4 (such as exenatide and liraglutide) or DPP-4 inhibitors (e.g. some gliptins) as a promising new therapy in NAFLD for their ability in modulating fatty acid oxidation, decreasing lipogenesis, and improving hepatic glucose metabolism [52].

\section{Other pharmacological agents}

There are not evidences about the rational for the use of lipid-lowering agents, angiotensin-converting enzyme (ACE) inhibitors, and angiotensin-receptor blockers (ARBs) in treatment of pediatric NAFLD [1].

\section{Discussions}

Data here reviewed confirm that weight loss and/or lifestyle changes remain the first choice treatment for adult and pediatric NAFLD, as well. Management strategy for an obese child with NAFLD should try to attain a gradual weight reduction by a multidisciplinary and long term therapeutic program combining diet and physical exercise. Diet prescription should be part of a wellstructured family-based program to enhance selfmotivation and success with diet. Physical exercise should be encouraged at all levels, since it might allow better metabolic control, weight reduction or even only its simple stability. Lack of compliance however remains a relevant problem, and dietary therapy for obesity generally fails to achieve its desired target. There is mounting indication that the high rate of relapse from weight loss during dietary therapy occurs because of complex compensatory biological adaptations and is not caused simply by lack of discipline and will power [2]. 
Disappointing results obtained also by the current standard pharmacological treatments (antioxidants and insulin-sensitizers) in the large multicenter TONIC study [23] call for careful re-thinking on the strategies hitherto pursued for the treatment of NAFLD, both in adults and in children. Future studies will probably need resorting to combined tailored treatments, targeting more than one pathogenetic mechanism.

The recent growing body of evidence on the role of gut microbiota and gut-liver-axis both in provoking or worsening obesity itself and/or its related complication including NAFLD and NASH, calls for robust well designed studies better focusing the mechanisms controlling possible derangements of the numerous metabolic, toxic, and immunological actors participating to the gut-liver -axis. At present gut microbiota modulation by probiotics starts appearing as the most promising tool due to their safety, tolerability and efficacy, at least in the preliminary pilot studies hitherto published $[34,45]$.

\section{Abbreviations}

ALT: Alanine aminotransferase; DPP-4: Dipeptidyl peptidase-4; FFA: Free fatty acids; FXR: Farnesoid X receptor; GLP-1: Glucagon-like peptid-1; IR: Insulin resistance; NAFLD: Non alcoholic fatty liver disease; NASH: Non alcoholic steatohepatitis; PAMPs: Pathogen-associated molecular patterns; PUFAs: Polyunsaturated fatty acids; ROS: Reactive oxygen substances; SIBO: Small intestinal bacterial overgrowth; TLR: Toll-like receptor; UDCA: Ursodeoxycholic acid.

\section{Competing interests}

Authors certificate that there are no conflict of interest.

Authors certificate that there are no financial competing interests.

\section{Authors' contributions}

PV and SL supervised the study and the final MS draft; GP, IDM, RDA prepared bibliographical background; CP, MS, and GP screened the papers retrieved by literature search. All worked on the draft MS and contributed to the text discussion. All authors read and approved the final manuscript.

\section{Author details}

${ }^{1}$ Chair of Pediatrics, Medical School of the University of Salerno, Salerno, Italy, and ELFID, Naples, Italy. ${ }^{2}$ Department of Pediatrics, University of Naples "Federico II", Naples, Italy.

Received: 17 September 2012 Accepted: 12 October 2012 Published: 17 October 2012

\section{References}

1. Chalasani N, Younossi Z, Lavine JE, Diehl AM, Brunt EM, Cusi K, Charlton M, Sanyal AJ: American Gastroenterological Association; American Association for the Study of Liver Diseases; American College of Gastroenterology: The diagnosis and management of non-alcoholic fatty liver disease: practice guideline by the American Gastroenterological Association, American Association for the Study of Liver Diseases, and American College of Gastroenterology. Gastroenterology 2012, 142:1592-1609.

2. Mark AL: Dietary therapy for obesity: an emperor with no clothes. Hypertension 2008, 51:1426-1434.

3. Socha P, Horvath A, Vajro P, Dziechciarz P, Dhawan A, Szajewska H: Pharmacological interventions for nonalcoholic fatty liver disease in adults and in children: a systematic review. J Pediatr Gastroenterol Nutr 2009, 48:587-596.

4. Shah K, Stufflebam A, Hilton TN, Sinacore DR, Klein S, Villareal DT: Diet and exercise interventions reduce intrahepatic fat content and improve insulin sensitivity in obese older adults. Obesity (Silver Spring) 2009, 17:2162-2168.

5. Reinehr T, Schmidt C, Toschke AM, Andler W: Lifestyle intervention in obese children with non-alcoholic fatty liver disease: 2-year followupstudy. Arch Dis Child 2009, 94:437-442.

6. Koot $\mathrm{BG}$, van der Baan-Slootweg $\mathrm{OH}$, Tamminga-Smeulders $\mathrm{CL}$, Rijcken $\mathrm{TH}$, Korevaar JC, van Aalderen WM, Jansen PL, Benninga MA: Lifestyle intervention for non-alcoholic fatty liver disease: prospective cohort study of its efficacy and factors related to improvement. Arch Dis Child 2011, 96:669-674.

7. Vajro P, Fontanella A, Perna C, Orso G, Tedesco M, De Vincenzo A: Persistent hyperaminotransferasemia resolving after weight reduction in obese children. J Pediatr 1994, 125:239-241.

8. Franzese A, Vajro P, Argenziano A, Puzziello A, lannucci MP, Saviano MC, Brunetti F, Rubino A: Liver involvement in obese children. Ultrasonography and liver enzyme levels at diagnosis and during follow-up in an Italian population. Dig Dis Sci 1997, 42:1428-1432.

9. Abdelmalek MF, Suzuki A, Guy C, Unalp-Arida A, Colvin R, Johnson RJ, Diehl AM, Nonalcoholic Steatohepatitis Clinical Research Network: Increased fructose consumption is associated with fibrosis severity in patients with nonalcoholic fatty liver disease. Hepatology 2010, 51:1961-1971.

10. ZelberSagi S, Nitzan-Kaluski D, Goldsmith R, Webb M, Blendis L, Halpern Z, Oren R: Long term nutritional intake and the risk for Non-alcoholic fatty liver disease (NAFLD) a population based study. J Hepatol 2007, 47:711-717.

11. Nobili V, Bedogni G, Alisi A, Pietrobattista A, Risé P, Galli C, Agostoni C: Docosahexaenoic acid supplementation decreases liver fat content in children with non-alcoholic fatty liver disease: double-blind randomized controlled clinical trial. Arch Dis Child 2011, 96:350-353.

12. Huang JS, Barlow SE, Quiros-Tejeira RE, Scheimann A, Skelton J, Suskind D, Tsai P, Uko V, Warolin JP, Xanthakos SA, The NASPGHAN Obesity Task Force: Consensus Statement: Childhood Obesity for Pediatric Gastroenterologists. J Pediatr Gastroenterol Nutr 2012, Epub ahead of print.

13. ESPGHAN Committee on Nutrition, Agostoni C, Braegger C, Decsi T, Kolacek S, Koletzko B, Mihatsch W, Moreno LA, Puntis J, Shamir R, Szajewska H, Turck $D$, van Goudoever J: Role of dietary factors and food habits in the development of childhood obesity: a commentary by the ESPGHAN Committee on Nutrition. J Pediatr Gastroenterol Nutr 2011, 52:662-669.

14. Lin YC, Chang PF, Yeh SJ, Liu K, Chen HC: Risk factors for liver steatosis in obese children and adolescents. Pediatr Neonatol 2010, 51:149-154.

15. McCurdy LE, Winterbottom KE, Mehta SS, Roberts JR: Using nature and outdoor activity to improve children's health. Curr Probl Pediatr Adolesc Health Care 2010, 40:102-117.

16. Hannukainen JC, Nuutila P, Borra R, Kaprio J, Kujala UM, Janatuinen T, Heinonen OJ, Kapanen J, Viljanen T, Haaparanta M, Rönnemaa T, Parkkola R, Knuuti J, Kalliokoski KK: Increased physical activity decreases hepatic free fatty acid uptake: a study in human monozygotic twins. J Physiol 2007, 578:347-358.

17. Tamura Y, Tanaka Y, Sato F, Choi JB, Watada H, Niwa M, Kinoshita J, Ooka A, Kumashiro N, Igarashi Y, Kyogoku S, Maehara T, Kawasumi M, Hirose T, Kawamori R: Effects of diet and exercise on muscle and liver intracellular lipid contents and insulin sensitivity in type 2 diabetic patients. J Clin Endocrinol Metab 2005, 90:3191-3196.

18. Van Der Heijden GJ, Wang ZJ, Chu Z, Toffolo G, Manesso E, Sauer PJ, Sunehag AL: Strength exercise improves muscle mass and hepatic insulin sensitivity in obese youth. Med Sci Sports Exerc 2010, 42:1973-1980.

19. Baños RM, Cebolla A, Botella C, García-Palacios A, Oliver E, Zaragoza I, Alcaniz M: Improving Childhood Obesity Treatment Using New Technologies: The ETIOBE System. Clin Pract Epidemiol Ment Health 2011, 7:62-66.

20. Mandato C, Lucariello S, Licenziati MR, Franzese A, Spagnuolo MI, Ficarella R, Pacilio M, Amitrano M, Capuano G, Meli R, Vajro P: Metabolic, hormonal, oxidative, and inflammatory factors in pediatric obesity related liver disease. J Pediatr 2005, 147:62-66.

21. Schwimmer JB, Middleton MS, Deutsch R, Lavine JE: A phase 2 clinical trial of metformin as a treatment for non-diabetic paediatric non-alcoholic steatohepatitis. Aliment Pharmacol Ther 2005, 21:871-879.

22. Nadeau KJ, Ehlers LB, Zeitler PS, Love-Osborne K: Treatment of nonalcoholic fatty liver disease with metformin versus lifestyle intervention in insulin-resistant adolescents. Pediatr Diabetes 2009, 10:5-13.

23. Lavine JE, Schwimmer JB, Van Natta ML: Effect of Vitamin E or Metformin for Treatment of Nonalcoholic Fatty Liver Disease in Children and 
Adolescents: The TONIC Randomized Controlled Trial. JAMA 2011, 305:1659-1668.

24. Lavine JE: Vitamin E treatment of nonalcoholic steatohepatitis in children: a pilot study. J Pediatr 2000, 136:734-738.

25. Vajro P, Mandato C, Franzese A, Ciccimarra E, Lucariello S, Savoia M, Capuano G, Migliaro F: Vitamin E treatment in pediatric obesity-related liver disease: a randomized study. J Pediatr Gastroenterol Nutr 2004, 38:48-55.

26. Wang CL, Liang L, Fu JF, Zou CC, Hong F, Xue JZ, Lu JR, Wu XM: Effect of lifestyle intervention on non-alcoholic fatty liver disease in Chinese obese children. World J Gastroenterol 2008, 14:1598-1602.

27. Nobili V, Manco M, Devito R, Di Ciommo V, Comparcola D, Sartorelli MR, Piemonte F, Marcellini M, Angulo P: Lifestyle intervention and antioxidant therapy in children with nonalcoholic fatty liver disease: a randomized, controlled trial. Hepatology 2008, 48:119-128.

28. Dohil R, Schmeltzer S, Cabrera BL, Wang T, Durelle J, Duke KB, Schwimmer $J B$, Lavine JE: Enteric-coated cysteamine for the treatment of paediatric non-alcoholic fatty liver disease. Aliment Pharmacol Ther 2011, 33:1036-44.

29. Cysteamine Bitartrate Delayed-Release for the Treatment of NAFLD in Children (CyNCh). http://clinicaltrials.gov.

30. Trauner M, Claudel T, Fickert P, Moustafa T, Wagner M: Bile acids as regulators of hepatic lipid and glucose metabolism. Dig Dis 2010, 28:220-224

31. Vajro P, Franzese A, Valerio G, lannucci MP, Aragione N: Lack of efficacy of ursodeoxycholic acid for the treatment of liver abnormalities in obese children. J Pediatr 2000, 136:739-743.

32. Diehl AM: Hepatic complications of obesity. Gastroenterol Clin North Am 2010, 39:57-68.

33. Volk ML: Risks of using ursodeoxycholic acid to treat primary sclerosing cholangitis. Gastroenterology 2011, 14:2267-2268.

34. Vajro P, Mandato C, Licenziati MR, Franzese A, Vitale DF, Lenta S, Caropreso M, Vallone G, Meli R: Effects of Lactobacillus rhamnosus strain GG in pediatric obesity-related liver disease. J Pediatr Gastroenterol Nutr 2011, 52:740-743.

35. Li W, Zheng L, Sheng C, Cheng X, Qing L, Qu S: Systematic review on the treatment of pentoxifylline in patients with non-alcoholic fatty liver disease. Lipids Health Dis 2011, 10:49.

36. Fuchs M: Non-alcoholic Fatty liver disease: the bile Acid-activated farnesoid $\mathrm{x}$ receptor as an emerging treatment target. J Lipids 2012, 2012:934396.

37. Musso G, Gambino R, Cassader M: Gut microbiota as a regulator of energy homeostasis and ectopic fat deposition: mechanisms and implications for metabolic disorders. Curr Opin Lipidol 2010, 21:76-83.

38. Kootte RS, Vrieze A, Holleman F, Dallinga-Thie GM, Zoetendal EG, de Vos WM, Groen AK, Hoekstra JB, Stroes ES, Nieuwdorp M: The therapeutic potential of manipulating gut microbiota in obesity and type 2 diabetes mellitus. Diabetes Obes Metab 2012, 14:112-120.

39. Wigg AJ, Roberts-Thomson IC, Dymock RB, McCarthy PJ, Grose RH, Cummins AG: The role of small intestinal bacterial overgrowth, intestinal permeability, endotoxaemia, and tumour necrosis factor alpha in the pathogenesis of non-alcoholic steatohepatitis. Gut 2001, 48:206-211.

40. Schrezenmeir J, de Vrese M: Probiotics, prebiotics, and synbioticsapproaching a definition. Am J Clin Nutr 2001, 73(2 Suppl):361S-364S.

41. Cani PD, Bibiloni R, Knauf C, Waget A, Neyrinck AM, Delzenne NM, Burcelin $\mathrm{R}$ : Changes in gut microbiota control metabolic endotoxemia-induced inflammation in high fat diet-induced obesity and diabetes in mice. Diabetes 2008, 57:1470-1481.

42. Esposito E, lacono A, Bianco G, Autore G, Cuzzocrea S, Vajro P, Canani RB, Calignano A, Raso GM, Meli R: Probiotics reduce the inflammatory response induced by a high-fat diet in the liver of young rats. $J$ Nutr 2009, 139:905-911

43. Loguercio C, Federico A, Tuccillo C, Terracciano F, D'Auria MV, De Simone C, Del Vecchio Blanco C: Beneficial effects of a probiotic VSL\#3 on parameters of liver dysfunction in chronic liver diseases. $J$ Clin Gastroenterol 2005, 39:540-543.

44. Lirussi F, Mastropasqua E, Orando S, Orlando R: Probiotics for non-alcoholic fatty liver disease and/or steatohepatitis. Cochrane Database Syst Rev 2007, 1. CD005165.

45. Aller $\mathrm{R}$, De Luis DA, Izaola O, Conde R, Gonzalez Sagrado M, Primo D, De La Fuente B, Gonzalez J: Effect of a probiotic on liver aminotransferases in nonalcoholic fatty liver disease patients: a double blind randomized clinical trial. Eur Rev Med Pharmacol Sci 2011, 9:1090-1095.
46. Parnell JA, Reimer RA: Prebiotic fiber modulation of the gut microbiota improves risk factors for obesity and the metabolic syndrome. Gut Microbes 2012, 1:29-34

47. Masterton GS, Plevris JN, Hayes PC: Review article: omega-3 fatty acids - a promising novel therapy for non-alcoholic fatty liver disease. Aliment Pharmacol Ther 2010, 31:679-692.

48. Weiner RA: Surgical treatment of non-alcoholic steatohepatitis and nonalcoholic fatty liver disease. Dig Dis 2010, 28:274-279.

49. Fullmer MA, Abrams SH, Hrovat K, Mooney L, Scheimann AO, Hillman JB, Suskind DL, National Association of Children's Hospitals and Related Institutions; North American Society of Pediatric Gastroenterology, Hepatology, and Nutrition: Nutritional strategy for adolescents undergoing bariatric surgery: report of a working group of the Nutrition Committee of NASPGHAN/NACHRI. J Pediatr Gastroenterol Nutr 2012, 54:125-135.

50. Miura K, Seki E, Ohnishi H, Brenner DA: Role of toll-like receptors and their downstream molecole in the development of nonalcoholic Fatty liver disease. Gastroenterol Res Pract 2010, 2010:362847.

51. Kanzler H, Barrat FJ, Hessel EM, Coffman RL: Therapeutic targeting of innate immunity with Toll-like receptor agonists and antagonists. Nat Med 2007, 13:552-559.

52. Lee J, Hong SW, Rhee EJ, Lee WY: GLP-1 Receptor Agonist and NonAlcoholic Fatty Liver Disease. Diabetes Metab J 2012, 36:262-267.

doi:10.1186/1824-7288-38-55

Cite this article as: Vajro et al:: Therapeutic options in pediatric non alcoholic fatty liver disease: current status and future directions. Italian Journal of Pediatrics 2012 38:55.

\section{Submit your next manuscript to BioMed Central and take full advantage of:}

- Convenient online submission

- Thorough peer review

- No space constraints or color figure charges

- Immediate publication on acceptance

- Inclusion in PubMed, CAS, Scopus and Google Scholar

- Research which is freely available for redistribution 